\title{
APPLICATIONS ON THE BOUNDARY BEHAVIOUR OF THE DERIVATIVE OF CONFORMAL MAPPING
}

\author{
SHATHA S. ALHILY ${ }^{1}$, VISHNU NARAYAN MISHRA ${ }^{2, *}$ \\ ${ }^{1}$ Department of Mathematics, College of Science, Mustansiriyah University, Baghdad, Iraq \\ shathamaths@uomustansiriyah.edu.iq,shathamaths@yahoo.co.uk \\ ${ }^{2}$ Department of Mathematics, Indira Gandhi National Tribal University, Lalpur, Amarkantak, Anuppur 484 \\ 887, India \\ *Corresponding author: vishnunarayanmishra@gmail.com, vnm@igntu.ac.in

\begin{abstract}
The objective of this research paper is to describe the behaviour of the boundary derivative of conformal maps from polygon domain onto unit disk through construct some an interesting cases, and its inverse maps. Moreover, we study the existence and finiteness the integrability of the derivative of conformal maps over an infinite sector $\mathbb{W}$.
\end{abstract}

\section{INTRODUCTION}

Let $\phi$ be a conformal map from $\mathbb{D} \subset \mathbb{C}$ onto a simply connected domain $\Omega$, with its inverse $\psi=\phi^{-1}: \Omega \longrightarrow \mathbb{D}$.

Brennan's conjecture states that, for all such $\phi$,

$$
\iint_{\mathbb{D}}\left|\phi^{\prime}\right|^{2-p} d x d y=\iint_{\Omega}\left|\psi^{\prime}\right|^{p} d x d y<\infty, \text { for } \frac{4}{3}<p<4 .
$$

In connection with this conjecture which is associated with estimating the integral means of derivatives of univalent functions, which is so related with the behaviour of the boundary derivative of conformal maps from polygon domain onto unit disk and the behaviour of the boundary of inverse maps

Received March $7^{\text {th }}, 2020$; accepted August $3^{\text {rd }}, 2020$; published August $2^{\text {nd }}, 2021$.

2010 Mathematics Subject Classification. 30B30.

Key words and phrases. conformal mapping; derivative of conformal maps; infinite sector; Polygon domain.

(C)2021 Authors retain the copyrights of their papers, and all open access articles are distributed under the terms of the Creative Commons Attribution License. 
An existence and finiteness the integrability of the derivative of conformal maps through constructing some interesting examples

when engages the infinite sector $\mathbb{W}$.

We started with basic definition of the polygonal domain and we present some typical examples to examine the boundary behaviour of the derivatives of conformal mapping of polygon to unit disk, as well as the boundary behaviour of the derivatives of the inverse maps.

In the following some preliminary on the boundary derivative of conformal maps, with basic definition of the polygonal domain that serves as motivation to the main results of this work to give a full support, the reader is referred to references $[2,3,7-9,11,12]$.

Definition 1.1 (Polygon). A polygon $P$ is usually defined as a collection of $n$ vertices $v_{1}, v_{2}, \ldots, v_{n}$ and $n$ edges $v_{1} v_{2}, v_{2} v_{3}, \ldots, v_{n-1} v_{n}, v_{n} v_{1}$ such that no pair of nonconsecutive edges share a point. We deviate from the usual practice by defining a polygon as the closed finite connected region of the plane bounded by these vertices and edges. The collection of vertices and edges will be referred to as the boundary of $P$, denoted by $\partial P$, a polygon of $n$ vertices will sometimes be called an n-gon.

Riemann mapping theorem [6]. guarantees the existence of a conformal map $\phi$ from polygonal domain $P \subset \mathbb{C}$ conformally onto the unit disk $\mathbb{D}(|w|<1)$, which can be extended continuously to the boundary ( cf. Carathéodory's theorem [9]). Worthy to mention that it is not yet possible to write down a simple formula for the conformal map from one region to another. Hence, in case of a map from the upper half-plane or unit disk $\mathbb{D}$ to a polygon, then Schwarz-Christoffel formula [5] allows to compute the conformal map $\phi$ defined as follows:

Consider $\phi: P \longrightarrow \mathbb{D}$, be a conformal mapping, where $\partial P$ be a circular arc or straight line segment $\gamma$, normalized by the conditions $\phi\left(z_{0}\right)=0$ and $\phi^{\prime}\left(z_{0}\right)>0$ ( where $z_{0}$ is some point in $P$ ), and observe that $\phi$ maps $\gamma$ onto an arc $\hat{\gamma}$ of the unit circle $|w|=1$ with $\psi(w)=\phi^{-1}(z): \mathbb{D} \longrightarrow P$.

$$
\phi(z)=A+C \int \prod_{k=1}^{z}\left(\zeta-z_{k}\right)^{\alpha_{k}-1} d \zeta
$$

where $A$ and $C$ are suitably chosen constants.

So,we deliberately construct $\phi$ as the composition of one Schwarz-Christoffel map from $P$ into upper half-plane (by applying Schwarz-Christoffel transformation) and another map of the upper half-plane to unit disk, in the examples (2.1), (2.2) where $\phi$ maps the $P$ to unit disk.

\footnotetext{
The term "polygon" is often modified by "simple" to distinguish it from polygons that cross themselves, Simple polygons are also called Jordan polygons, because such a polygon divides the plane into two regions. The boundary of a polygon is a "Jordan curve" : it separates the plane into two disjoint regions, the interior and the exterior of the polygon.
} 
This technique could help to study the behavior of conformal mapping by estimating some quantities which belong to interior upper half-plane, which in turn will help to analyze the boundary behaviour of the derivative of this map.

\section{Main Results}

In the light of reported above, we discussed the theoretical underpinnings of the behaviour of the boundary derivative of conformal maps through of some main results obtained that have been yielded useful information in this regards. (cf. [1])

Theorem 2.1. The derivative of the conformal mapping defined on Rectangular domain to the Unit disk is bounded but the derivative of the inverse maps is unbounded.

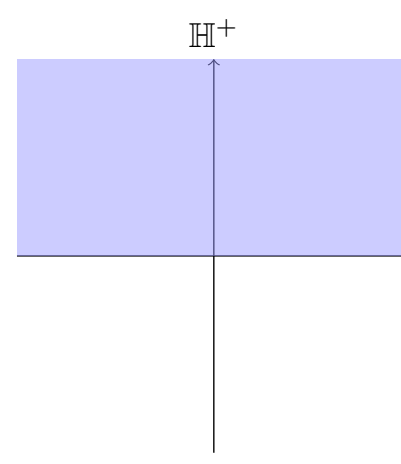

$$
\frac{\zeta=\operatorname{sn} z}{z=\operatorname{arcsn}(\zeta, k)}
$$
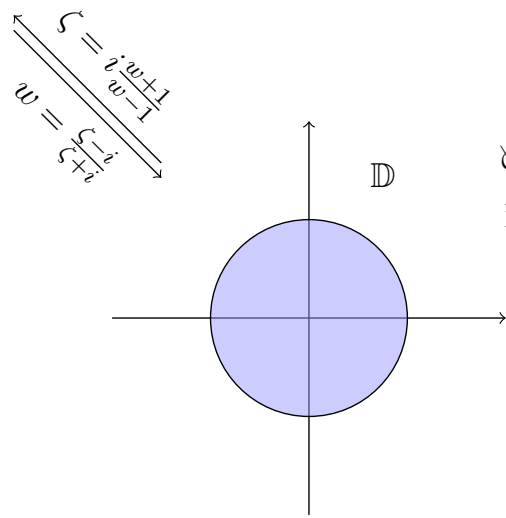

unit disk
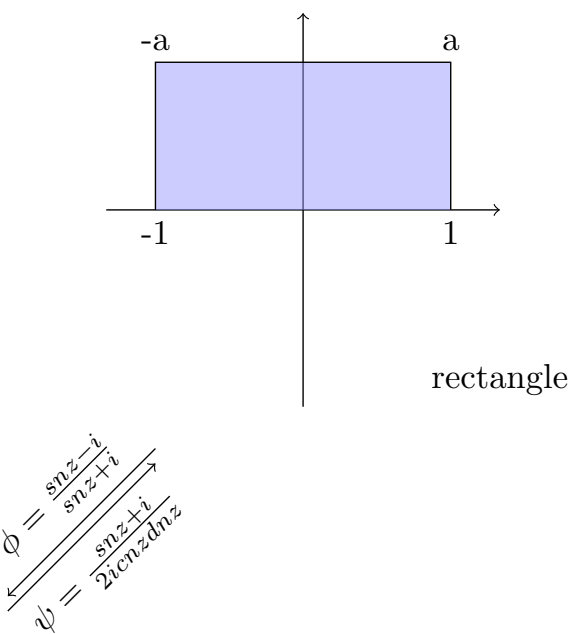

(2)

FiguRE 1. Conformal mappings from rectangular domain onto unit disk

Proof. Map the interior of the rectangular domain with vertices at points $z_{1}=1, z_{2}=-1, z_{3}=-a$, and $z_{4}=a$ with $a>1$ into upper half plane needs to define Schwarz- Christoffel transformtion which maps $\mathbb{H}^{+}$ 
into rectangle as follows:

$$
\begin{aligned}
& z=A+B \int_{\zeta_{o}}^{\zeta} \frac{d s}{(s-1)^{\frac{1}{2}}(s+1)^{\frac{1}{2}}(s-a)^{\frac{1}{2}}(s+a)^{\frac{1}{2}}} \\
& z=A+B \int_{\zeta_{o}}^{\zeta} \frac{d s}{\left(s^{2}-1\right)^{\frac{1}{2}}\left(s^{2}-a^{2}\right)^{\frac{1}{2}}}
\end{aligned}
$$

Suppose $A=0$ and $B=1$ for convenience.

$$
z=\int_{\zeta_{o}}^{\zeta} \frac{d s}{\sqrt{\left(s^{2}-1\right)\left(s^{2}-a^{2}\right)}}
$$

Suppose $\zeta_{o}=0$ and let $a=\frac{1}{k}$, then the integral (2.10) is transformed to

$$
\begin{gathered}
z=\int_{\zeta_{o}}^{\zeta} \frac{d s}{\sqrt{\left(s^{2}-1\right)\left(s^{2}-\left(\frac{1}{k}\right)^{2}\right)}}=k \int_{0}^{\zeta} \frac{d s}{\sqrt{\left(s^{2}-1\right)\left(k^{2} s^{2}-1\right)}} \\
z=k \int_{0}^{\zeta} \frac{d s}{\sqrt{\left(1-s^{2}\right)\left(1-k^{2} s^{2}\right)}}
\end{gathered}
$$

The integral (2.2) is called an elliptic integral of first kind and $k$ is a modulus of the elliptic integral with $0<k<1$, denote by

$$
z=s n^{-1}(\zeta, k)
$$

The inverse mapping of integral (2.2) is known as the Jacobi elliptic function denoted by

$$
\begin{aligned}
\zeta & =s n(z, k) . \\
\Rightarrow \phi & =\frac{\zeta-i}{\zeta+i}=\frac{s n z-i}{s n z+i}: \text { Rectangular domain } \longrightarrow \mathbb{D} \\
\phi^{\prime} & =\frac{(s n z+i)(c n z d n z)-(s n z-i)(d n z)}{(s n z+i)^{2}}=\frac{2 i c n z d n z}{(s n z+i)^{2}},
\end{aligned}
$$

such that, $d n z=\sqrt{1-k^{2} s n^{2} z}, c n z=\sqrt{1-s n^{2} z}$.

$$
\begin{aligned}
&\left|\phi^{\prime}\right|=\left|\frac{2 i c n z d n z}{(s n z+i)^{2}}\right|=\frac{2|c n z d n z|}{|s n z+i|^{2}} \\
& s n z \in H^{+} \Rightarrow s n z+i \in H^{+} \text {that is, }|s n z+i| \geq 1 \Rightarrow \frac{1}{|s n z+i|} \leq 1 \\
&|c n z d n z|=\left|\sqrt{1-k^{2} s n^{2} z \mid}\right| \sqrt{1-s n^{2} z \mid} \\
&\left|1-k^{2} s n^{2} z\right| \leq 1+|s n z|^{2} \\
&\left|1-s n^{2} z\right| \leq 1+|k \operatorname{snz}|^{2} \\
& \Rightarrow\left|\phi^{\prime}\right|=\frac{2|c n z d n z|}{|\operatorname{snz}+i|^{2}} \leq 2\left(1+|\operatorname{snz}|^{2}\right)\left(1+\mid k \text { snz }\left.\right|^{2}\right)
\end{aligned}
$$

$\Rightarrow\left|\phi^{\prime}\right|$ is bounded.

It remains to show that the inverse of the derivative of such function $\phi(z)$ is unbounded as follows: 


$$
\begin{gathered}
\psi=\phi^{-1}: \mathbb{D} \longrightarrow \text { rectangular domain } \\
\psi^{\prime}=\frac{1}{\phi^{\prime}}=\frac{(\text { snz }+i)^{2}}{2 i c n z d n z} \\
\Rightarrow \psi=\frac{(\text { snz }+i)^{2}}{2 i c n z d n z} \\
\left|\psi^{\prime}\right|=\frac{|\operatorname{snz} z i|^{2}}{2|c n z d n z|}
\end{gathered}
$$

$s n z \in H^{+} \Rightarrow(s n z+i) \in H^{+} \Rightarrow|s n z+i|^{2} \geq 1$.

$$
\begin{aligned}
\left|\psi^{\prime}\right| & =\frac{|\operatorname{snz}+i|^{2}}{2|\operatorname{cn} z d n z|} \\
& \geq \frac{1}{2|\operatorname{cnz} d n z|}
\end{aligned}
$$

$\Rightarrow\left|\psi^{\prime}\right|$ is unbounded as $|z| \longrightarrow 0$.

Theorem 2.2. The derivative of the conformal mapping defined on Triangular domain $\Delta$ to the unit disk is bounded but the derivative of the inverse maps is unbounded.

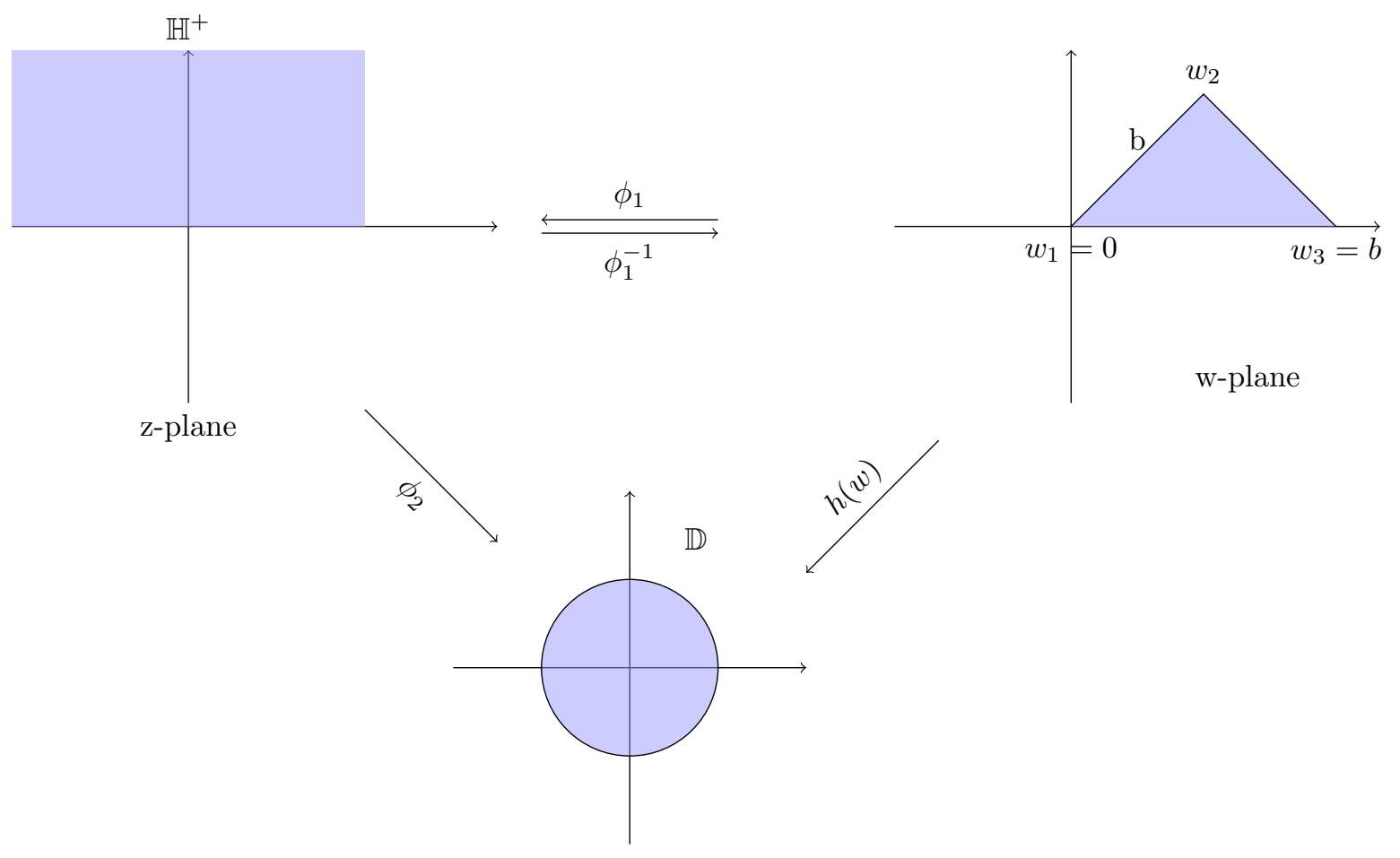

$\zeta$-plane

Figure 2. Conformal mappings from triangular domain onto unit disk 
Proof. To construct conformal mapping defined on triangle domain to unit disk $\mathbb{D}$. we have to define conformal mapping on triangular domain to upper half plane $H^{+}$and then define another mapping from $\mathbb{H}^{+}$ to unit disk $\mathbb{D}$ to achieve our aim.

To do so, First : we have to establish conformal mapping that maps upper half- plane $\mathbb{H}^{+}$onto triangular domain $\triangle$ by Schwarz-Christoffel transformation as follows:

Let

$$
\phi_{1}=A \int_{z_{o}}^{z}\left(s-x_{1}\right)^{-k_{1}}\left(s-x_{2}\right)^{-k_{2}} d s+B .
$$

such that $-k_{i}=\frac{\alpha_{i}}{\pi}-1 ; \forall i=1,2$ to be Schwars-Christoffel transformation that maps $\mathbb{H}^{+}$into the interior of the equilateral triangle $\triangle$ such that $\alpha_{i}=\frac{\pi}{3} ; \forall i=1,2,3$.

Now, by assisstance that $z_{o}=1, A=1$ and $B=0$, we obtain Schwars-Christoffel transformation defined as follows:

$$
\phi_{1}=\int_{1}^{z}(s+1)^{\frac{-2}{3}}(s-1)^{\frac{-2}{3}} d s
$$

Which maps $x_{1}=-1, x_{2}=1$ and $x_{3}=\infty$ into $\Delta w_{1} w_{2} w_{3}$ as follows:

(i) In case $z=1 \Rightarrow \phi_{1}(1)=0$; that is, $w_{1}=0$ in $H^{+}$.

(ii) In case $z=-1$, we have

$$
\phi_{1}(-1)=\int_{1}^{-1}(s+1)^{\frac{-2}{3}}(s-1)^{\frac{-2}{3}} d s=w_{2} .
$$

(iii) In case $z= \pm \infty$, we have

$$
\lim _{z \rightarrow \pm \infty} \phi_{1}=\int_{1}^{ \pm \infty}(s+1)^{\frac{-2}{3}}(s-1)^{\frac{-2}{3}} d s=w_{3} .
$$

To solve these integrals, let us consider first the equation (2.5) by choosing a path of the integration $z=x$ along the real axis in the positive sense, that is

$$
\begin{aligned}
& s-1=|s-1| e^{i \theta_{1}} \\
& s+1=|s+1| e^{i \theta_{2}} .
\end{aligned}
$$

The argument $\left(\theta_{1}+\theta_{2}\right)$ remains constant throughout integration from -1 to 1 since $(s+1)$ stays positive with zero argument, and $(s-1)$ has constant argument $\pi$. Therefore equation (2.5) yields

$$
\begin{gathered}
w_{2}=\phi_{1}(-1)=-\int_{-1}^{1}(x+1)^{\frac{-2}{3}}(1-x)^{\frac{-2}{3}}\left(-e^{-\frac{2 \pi i}{3}}\right) d s . \\
w_{2}=\phi_{1}(-1)=e^{\frac{\pi i}{3}} \int_{0}^{1} \frac{2 d x}{\left(1-x^{2}\right)^{\frac{-2}{3}}} .
\end{gathered}
$$


By letting $x=\sqrt{t}$ in equation (2.7), we obtain Beta function $\mathcal{B}\left(\frac{1}{2}, \frac{1}{3}\right)$ and

$$
\begin{aligned}
w_{2} & =\phi_{1}(-1)=e^{\frac{\pi i}{3}} \mathcal{B}\left(\frac{1}{2}, \frac{1}{3}\right) . \\
\Rightarrow w_{2} & =\mathbf{b} e^{\frac{\pi i}{3}} .
\end{aligned}
$$

where $\mathbf{b}$ is the value of $\mathcal{B}\left(\frac{1}{2}, \frac{1}{3}\right)$ Now, the vertex $w_{3}$ lies on the positive $u$ - axis.

So, $w_{3}$ must be represented by the boundary integral 1 to $\infty$ as follows,

$$
\begin{gathered}
w_{3}=\phi_{1}(\infty)=\int_{1}^{\infty}(x+1)^{\frac{-2}{3}}(1-x)^{\frac{-2}{3}} d x \\
w_{3}=\phi_{1}(\infty)=\int_{1}^{\infty} \frac{d x}{\left(x^{2}-1\right)^{\frac{-2}{3}}} .
\end{gathered}
$$

But $w_{3}$ is also represented by integral (2.4) when $z=-\infty$ along the negative real axis.

So,

$$
\begin{aligned}
w_{3}=\phi_{1}(-\infty) & =\int_{1}^{-\infty}(x+1)^{\frac{-2}{3}}(1-x)^{\frac{-2}{3}} d x \\
& =\int_{1}^{-1}(x+1)^{\frac{-2}{3}}(1-x)^{\frac{-2}{3}} e^{\frac{-2 \pi i}{3}} d x+\int_{-1}^{-\infty}(x+1)^{\frac{-2}{3}}(1-x)^{\frac{-2}{3}} e^{\frac{-4 \pi i}{3}} d x . \\
w_{3} & =w_{1}+e^{\frac{-\pi i}{3}} \int_{1}^{\infty} \frac{d x}{\left(x^{2}-1\right)^{\frac{2}{3}}} \\
\Rightarrow w_{3} & =w_{1}+e^{\frac{-\pi i}{3}} w_{3} .
\end{aligned}
$$

Solving (2.9) for $w_{3}$ we obtain:

$$
\begin{aligned}
\Rightarrow w_{3}-e^{\frac{-\pi i}{3}} w_{3} & =w_{1} . \\
w_{3}\left(1-e^{\frac{-\pi i}{3}}\right) & =b e^{\frac{-\pi i}{3}} \\
w_{3} & =b ; \text { since }\left(1-e^{\frac{-\pi i}{3}}\right)=e^{\frac{-\pi i}{3}} .
\end{aligned}
$$

In the end, we found the conformal mapping that maps upper half- plane $\mathbb{H}^{+}$onto triangular domain $\triangle$.

It is known that $\phi_{2}=\frac{z-i}{z+i}$ maps upper half- plane $\mathbb{H}^{+}$onto unit disk $\mathbb{D}$.

Hence, we have

$$
\begin{aligned}
\phi_{1} & =\int_{1}^{z}\left(s^{2}-1\right)^{\frac{-2}{3}} d s: \text { upper half plane } \mathbb{H}^{+} \longrightarrow \text { triangular domain } \triangle \\
\text { and } \phi_{2} & =\frac{z-i}{z+i}: \quad \text { upper half- plane } \mathbb{H}^{+} \rightarrow \text { unit disk } \mathbb{D} \text {. }
\end{aligned}
$$

Let

$$
h(w) \text { : triangular domain } \Delta \longrightarrow \text { unit disk } \mathbb{D} \text {. }
$$


defined as follows:

$$
\begin{aligned}
h(w) & =\left(\phi_{2} \circ \phi_{1}^{-1}\right)(w)=\phi_{2}\left(\phi_{1}^{-1}\right)(w) \\
h^{\prime}(w) & =\phi_{2}^{\prime}\left(\phi_{1}^{-1}\right)\left(\phi_{1}^{\prime-1}\right) \\
& =\left(\frac{\phi_{1}^{-1}-i}{\phi_{1}^{-1}+i}\right)^{\prime}\left(\frac{1}{\phi_{1}^{\prime}}\right) \\
& =\frac{\left(\phi_{1}^{-1}+i\right)\left(\phi_{1}^{\prime-1}\right)-\left(\phi_{1}^{-1}-i\right)\left(\phi_{1}^{\prime-1}\right)}{\left(\phi_{1}^{-1}+i\right)^{2}} \frac{1}{\phi_{1}^{\prime}} . \\
\Rightarrow h^{\prime}(w) & =\frac{2 i\left(z^{2}-1\right)^{\frac{2}{3}}}{\left(\phi_{1}^{-1}+i\right)^{2}}\left(z^{2}-1\right)^{\frac{2}{3}} \\
& =\frac{2 i\left(z^{2}-1\right)^{\frac{4}{3}}}{\left(\phi_{1}^{-1}+i\right)^{2}} . \\
\left|h^{\prime}(w)\right| & =\left|\frac{2 i\left(z^{2}-1\right)^{\frac{4}{3}}}{\left(\phi_{1}^{-1}+i\right)^{2}}\right|=\frac{2 i\left|z^{2}-1\right|^{\frac{4}{3}}}{\left|\phi_{1}^{-1}+i\right|^{2}},
\end{aligned}
$$

where $\left(\phi_{1}^{-1}\right)^{\prime}=\frac{1}{\phi_{1}^{\prime}}=\left(z^{2}-1\right)^{\frac{2}{3}}$ and $\phi_{1}^{-1} \in \mathbb{H}^{+}$, so that $\left(\phi_{1}^{-1}+i\right) \in \mathbb{H}^{+}$.

This implies to

$$
\begin{aligned}
& \left|\phi_{1}^{-1}+i\right|^{2} \geq 1 \Rightarrow \frac{1}{\left|\phi_{1}^{-1}+i\right|^{2}} \leq 1 \\
& \Rightarrow\left|h^{\prime}(w)\right| \leq 2\left(|z|^{2}+1\right)^{\frac{4}{3}} .
\end{aligned}
$$

$\left|h^{\prime}(w)\right|$ is bounded.

What remains is to prove that the inverse of the derivative of $h(w)$ is unbounded.

Note that; $\phi_{2}^{-1}=-i \frac{\zeta+1}{\zeta-1}$ is the Möbius transformation, it maps the unit disk $\mathbb{D}$ to upper half-plane $\mathbb{H}^{+}$, thus we have

$$
\left(\phi_{2}^{-1}\right)^{\prime}=\frac{(\zeta-1)(-i)-(-i \zeta-i)}{(\zeta-1)^{2}}=\frac{2 i}{(\zeta-1)^{2}}
$$

We use Schwarz-Christoffel transformation for mapping $\mathbb{H}^{+}$into triangular domain $\triangle$.

$$
\phi_{1}=\int_{1}^{z}\left(s^{2}-1\right)^{\frac{-2}{3}} d s
$$


Therefore,

$$
\begin{aligned}
& h^{-1}(\zeta)=\left(\phi_{1} \circ \phi_{2}^{-1}\right)(\zeta): \mathbb{D} \longrightarrow \triangle \\
& \left(h^{-1}(\zeta)\right)^{\prime}=\phi_{1}^{\prime}\left(\phi_{2}^{-1}(\zeta)\right)\left(\phi_{2}^{-1}(\zeta)\right)^{\prime} \\
& =\left[\left(\phi_{2}^{-1}(\zeta)\right)^{2}-1\right]^{\frac{-2}{3}} \frac{2 i}{(\zeta-1)^{2}} . \\
& =\frac{2 i\left[-\left(\frac{\zeta+1}{\zeta-1}\right)^{2}-1\right]^{\frac{-2}{3}}}{(\zeta-1)^{2}} \\
& =\frac{2 i\left[\frac{-2 \zeta^{2}-2}{(\zeta-1)^{2}}\right]^{\frac{-2}{3}}}{(\zeta-1)^{2}} \text {. } \\
& =\frac{2 i}{(\zeta-1)^{2}\left[\frac{-2 \zeta^{2}-2}{(\zeta-1)^{2}}\right]^{\frac{-2}{3}}} \\
& =\frac{2 i}{(\zeta-1)^{2}\left(-2 \zeta^{2}-2\right)^{\frac{2}{3}}(\zeta-1)^{\frac{-4}{3}}} \\
& =\frac{2 i}{(\zeta-1)^{\frac{2}{3}}\left(-2 \zeta^{2}-2\right)^{\frac{2}{3}}} \text {. } \\
& \left|\left(h^{-1}(\zeta)\right)^{\prime}\right|=\left|\frac{2 i}{(\zeta-1)^{\frac{2}{3}}\left(-2 \zeta^{2}-2\right)^{\frac{2}{3}}}\right| . \\
& \left|\left(h^{-1}(\zeta)\right)^{\prime}\right|=\left|\frac{2}{(\zeta-1)^{\frac{2}{3}}\left(2 \zeta^{2}+2\right)^{\frac{2}{3}}}\right| \longrightarrow \infty \text { as } \zeta \longrightarrow 1 \text {. }
\end{aligned}
$$

Theorem 2.3. The derivative of the conformal mapping defined on crescent domain to unit disk is bounded but the derivative of the inverse maps is unbounded.

Proof. Compute the conformal mapping of a crescent domain onto unit disk by setting a sequence of functions as follows:

Let

$$
\begin{aligned}
& \phi_{1}=\frac{1}{z} \text { maps } C_{1} \rightarrow L_{1} \text { and } C_{2} \rightarrow L_{2} . \\
& \phi_{2}=\frac{i}{z} \text { rotates the stripe in the left plane onto stripe in the lower half plane. } \\
& \phi_{3}=\frac{4 \pi i}{z} \text { extends the stripe in the lower half plane } \mathbb{H}^{-} \text {between }-\pi,-2 \pi \\
& \phi_{4}=e^{\frac{4 i \pi}{z}} \text { maps the stripe in the lower half plane } \mathbb{H}^{-} \text {into } \mathbb{H}^{+} . \\
& \phi_{5}=\frac{e^{\frac{4 i \pi}{z}}-i}{e^{\frac{4 i \pi}{z}}+i} \text { maps the } \mathbb{H}^{+} \text {onto unit disk } \mathbb{D} .
\end{aligned}
$$




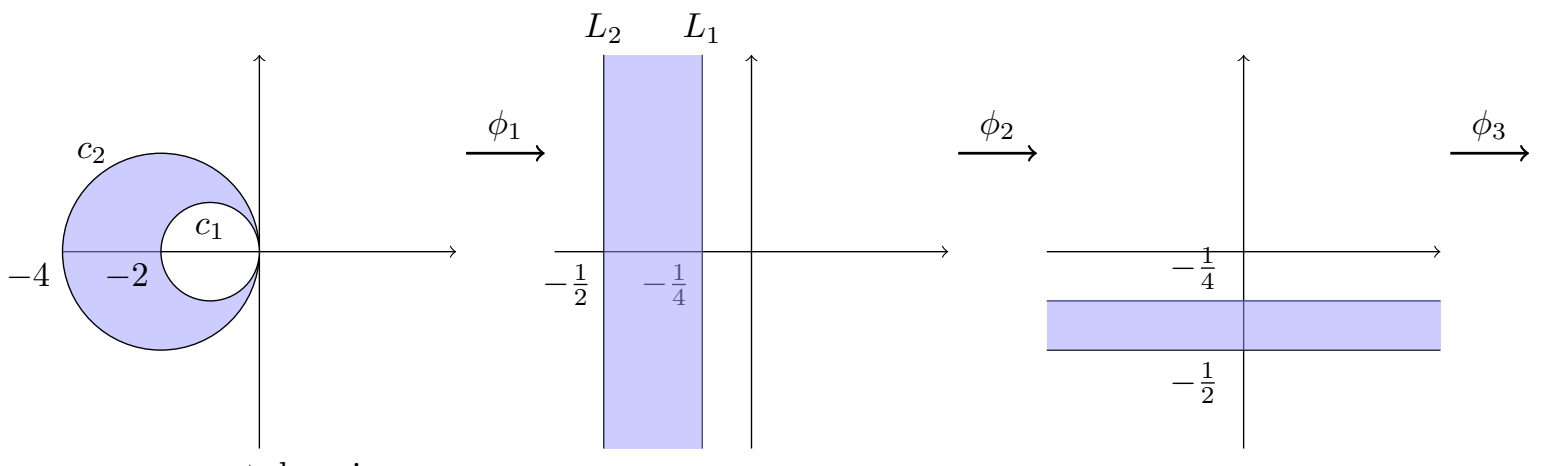

crescent domain
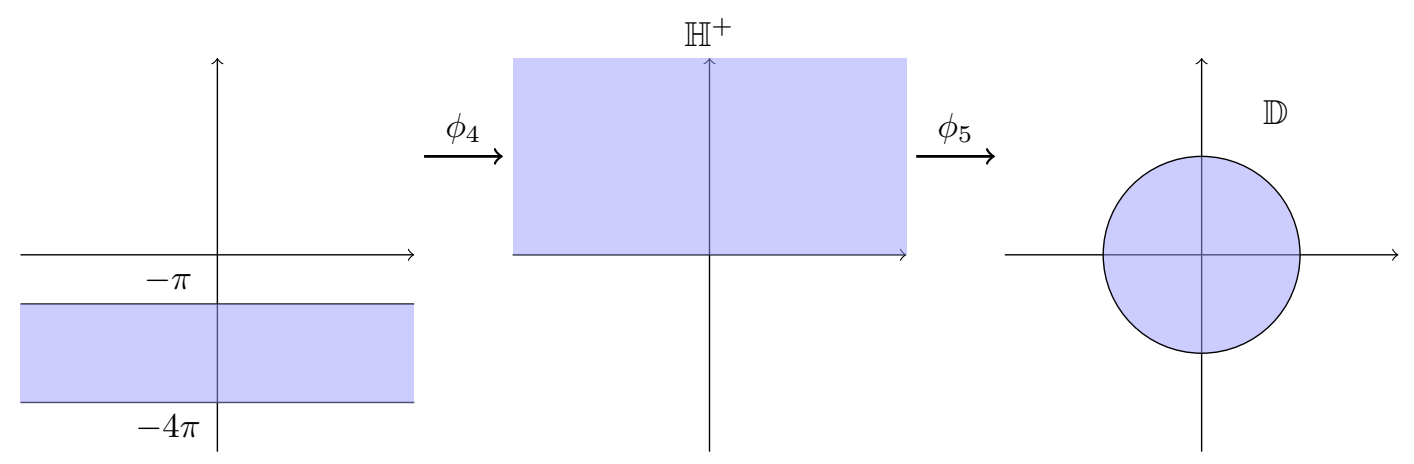

unit disk

FiguRE 3. Conformal mappings from crescent domain onto unit disk

So;

$$
\phi(z)=\frac{e^{\frac{4 i \pi}{z}}-i}{e^{\frac{4 i \pi}{z}}+i}: \text { crescent domain } \longrightarrow \text { unit disk } \mathbb{D} .
$$

Using short calculation we obtain

$$
\begin{aligned}
\phi^{\prime}(z) & =\frac{\left(e^{\frac{4 i \pi}{z}}+i\right)\left(\frac{-4 i \pi}{z^{2}} e^{\frac{4 i \pi}{z}}\right)-\left(e^{\frac{4 i \pi}{z}}-i\right)\left(\frac{-4 i \pi}{z^{2}} e^{\frac{4 i \pi}{z}}\right)}{\left(e^{\frac{4 i \pi}{z}}+i\right)^{2}} . \\
\Rightarrow \phi^{\prime}(z) & =\frac{\frac{8 \pi}{z^{2}} e^{\frac{4 i \pi}{z}}}{\left(e^{\frac{4 i \pi}{z}}+i\right)^{2}}=\frac{8 \pi e^{\frac{4 i \pi}{z}}}{z^{2}\left(e^{\frac{4 i \pi}{z}}+i\right)^{2}} \\
\left|\phi^{\prime}(z)\right| & =\frac{8 \pi\left|e^{\frac{4 i \pi}{z}}\right|}{|z|^{2}\left|e^{\frac{4 i \pi}{z}}+i\right|^{2}}
\end{aligned}
$$


Now;

$$
\begin{aligned}
\left|e^{\frac{4 i \pi}{z}}\right| & =1 \Rightarrow e^{\frac{4 i \pi}{z}} \in H^{+} \Rightarrow\left(e^{\frac{4 i \pi}{z}}+i\right) \in H^{+} \\
\Rightarrow\left|e^{\frac{4 i \pi}{z}}+i\right| & \geq 1 \Rightarrow\left|e^{\frac{4 i \pi}{z}}+i\right|^{2} \geq 1 \\
\Rightarrow \frac{1}{\left|e^{\frac{4 i \pi}{z}}+i\right|^{2}} & \leq 1 \\
\Rightarrow\left|\phi^{\prime}(z)\right| & =\frac{8 \pi\left|e^{\frac{4 i \pi}{z}}\right|}{|z|^{2}\left|e^{\frac{4 i \pi}{z}}+i\right|^{2}} \\
\leq & \frac{8 \pi}{|z|^{2}}
\end{aligned}
$$

Hence, $\left|\phi^{\prime}(z)\right|$ is bounded. We show that the inverse of the derivative of such function $\phi(z)$ is unbounded as follows:

$$
\begin{aligned}
\phi^{-1}(w) & =\psi(w): \mathbb{D} \longrightarrow \text { crescent domain. } \\
\psi & =\phi^{-1}=\frac{1}{\phi}=\frac{e^{\frac{4 \pi i}{z}}+i}{e^{\frac{4 \pi i}{z}}-i} \\
\Rightarrow \psi^{\prime} & =\frac{1}{\phi^{\prime}} \\
\Rightarrow \psi^{\prime} & =\frac{z^{2}\left(e^{\frac{4 i \pi}{z}}+i\right)}{8 \pi e^{\frac{4 i \pi}{z}}} \\
\Rightarrow\left|\psi^{\prime}\right| & =\frac{|z|^{2}\left|e^{\frac{4 i \pi}{z}}+i\right|}{8 \pi\left|e^{\frac{4 i \pi}{z}}\right|}
\end{aligned}
$$

It is known, $e^{\frac{4 i \pi}{z}} \in H^{+} \Rightarrow\left(e^{\frac{4 i \pi}{z}}+i\right) \in H^{+}$. Hence

$$
\begin{aligned}
\left|e^{\frac{4 i \pi}{z}}+i\right| & \geq 1 \&\left|e^{\frac{4 i \pi}{z}}\right|=1 \\
\Rightarrow\left|\psi^{\prime}\right| & =\frac{|z|^{2}\left|e^{\frac{4 i \pi}{z}}+i\right|}{8 \pi\left|e^{\frac{4 i \pi}{z}}\right|} \geq \frac{|z|^{2}}{8 \pi} .
\end{aligned}
$$

In the end, we obtain $\left|\psi^{\prime}\right|$ is unbounded.

Theorem 2.4. The derivative of the conformal mapping defined on Lens- shaped domain to the unit disk is bounded but the derivative of the inverse maps is unbounded.

Proof. Compute the conformal mapping a lens domain onto unit disk by setting a sequence of functions as follows: 

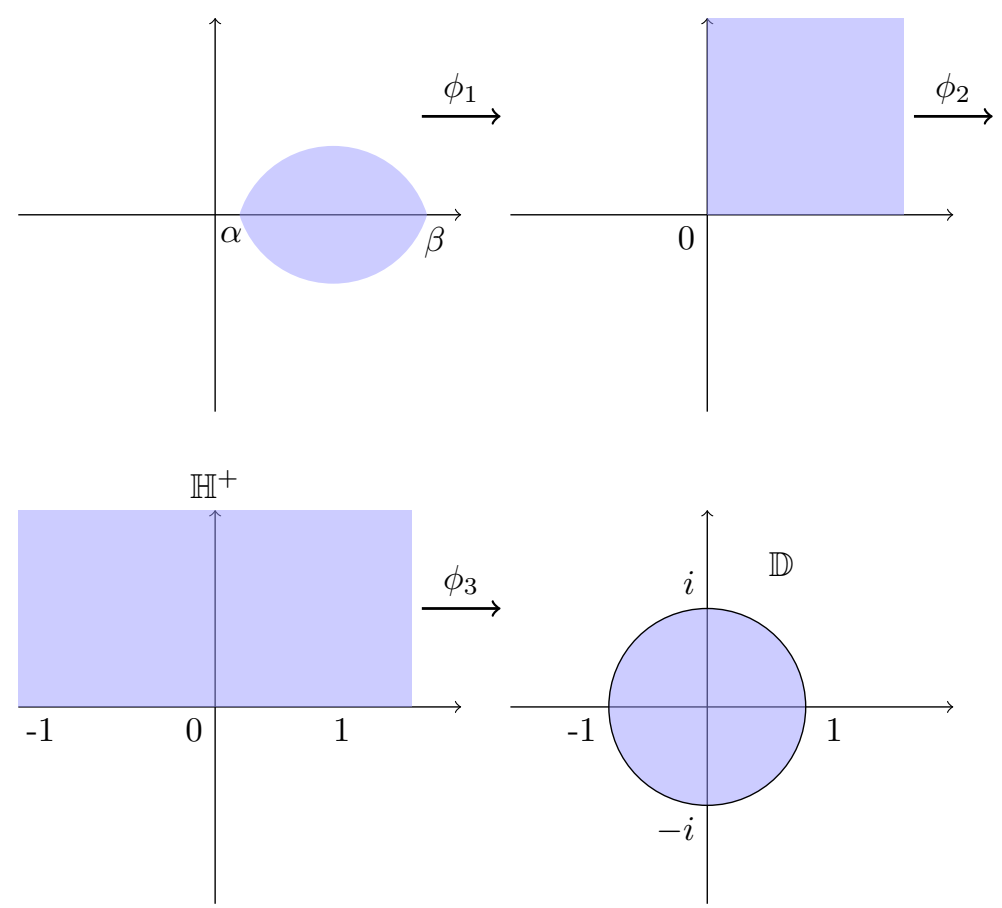

unit disk

Figure 4. Conformal mappings from lens domain onto unit disk

Let

$$
\begin{aligned}
& \phi_{1}=\frac{z-\alpha}{z-\beta} \text { maps Lens-shaped domain to the first quarter plane } \\
& \phi_{2}=z^{2} \text { maps the first quarter plane to the upper half- plane. } \\
& \phi_{3}=\frac{z^{2}-i}{z^{2}+i} \text { maps upper half plane } \mathbb{H}^{+} \text {to the unit disk } \mathbb{D} \text {. }
\end{aligned}
$$

So,

$$
\begin{aligned}
\phi(z) & =\frac{\left(\frac{z-\alpha}{z-\beta}\right)^{2}-i}{\left(\frac{z-\alpha}{z-\beta}\right)^{2}+i}: \text { Lens-shaped domain } \longrightarrow \text { unit disk } \mathbb{D} . \\
\Rightarrow \phi(z) & =\frac{(z-\alpha)^{2}-i(z-\beta)^{2}}{(z-\alpha)^{2}+i(z-\beta)^{2}}
\end{aligned}
$$

Therefore when

$$
\begin{aligned}
& z=\alpha \Rightarrow \phi(z)=-1 \text { in } \mathbb{D} \\
& z=\beta \Rightarrow \phi(z)=1 \text { in } \mathbb{D} .
\end{aligned}
$$


When

$$
\begin{aligned}
& (z-\alpha)^{2}=-(z-\beta)^{2} \\
& \Rightarrow \phi(z)=\frac{-(z-\beta)^{2}-i(z-\beta)^{2}}{-(z-\beta)^{2}+i(z-\beta)^{2}}=i \text { in } \mathbb{D} .
\end{aligned}
$$

In the end, if

$$
\begin{aligned}
& (z-\alpha)^{2}=(z-\beta)^{2} \\
\Rightarrow & \phi(z)=\frac{(z-\beta)^{2}-i(z-\beta)^{2}}{(z-\beta)^{2}+i(z-\beta)^{2}}=-i \text { in } \mathbb{D} . \\
\Rightarrow & \phi^{\prime}(z)=\frac{4 i(z-\beta)^{2}(z-\alpha)-4 i(z-\beta)(z-\alpha)^{2}}{\left[(z-\alpha)^{2}+i(z-\beta)^{2}\right]^{2}} \\
\Rightarrow & \left|\phi^{\prime}(z)\right|=\frac{4|\alpha-\beta||z-\beta||z-\alpha|}{(z-\alpha)^{4}+(z-\beta)^{4}} .
\end{aligned}
$$

where, $4|\alpha-\beta|=c ; c$ is a constant; $(\alpha<\beta)$.

Also,

$$
\begin{gathered}
|z-\beta| \leq|z|+|\beta|=M_{1} \\
\&|z-\alpha| \leq|z|+|\alpha|=M_{2} \\
\Rightarrow\left|\phi^{\prime}(z)\right| \leq \frac{M}{(z-\alpha)^{4}+(z-\beta)^{4}}
\end{gathered}
$$

$\Rightarrow\left|\phi^{\prime}(z)\right|$ is a bounded for every $z$ in Lens-shaped domain. Again, we can show that the inverse of the derivative of such function $\phi(z)$ is unbounded as follows:

$$
\begin{aligned}
\psi^{\prime} & =\frac{1}{\phi^{\prime}}=\frac{\left[(z-\alpha)^{2}+i(z-\beta)^{2}\right]^{2}}{4 i(z-\beta)^{2}(z-\alpha)-4 i(z-\beta)(z-\alpha)^{2}} \\
\Rightarrow\left|\psi^{\prime}\right| & =\frac{\left|(z-\alpha)^{2}+i(z-\beta)^{2}\right|^{2}}{4|(z-\beta)(z-\alpha)(\alpha-\beta)|} \\
\Rightarrow\left|\psi^{\prime}\right| & =\frac{(z-\alpha)^{4}+(z-\beta)^{4}}{4|z-\beta||z-\alpha||\alpha-\beta|} .
\end{aligned}
$$

If $z \longrightarrow \alpha$ or $z \longrightarrow \beta$, then $\left|\psi^{\prime}\right| \longrightarrow \infty$, so $\left|\psi^{\prime}\right|$ is unbounded.

The following examples show the integrability of the derivative of conformal maps on infinite sector $\mathbb{W}$ exists and is finite for some $p$ th-power integrable function $\phi$ when $\alpha=\frac{\pi}{n}$ is a number for some integer $n$.

Further details can be found in the books of Di Francesco [4] and of M. Stein [10].

Theorem 2.5. Let $\phi(z)$ be a conformal mapping defined on infinite sector $W$ for the angle $\alpha=\frac{\pi}{2}$ onto unit disk $\mathbb{D}$. 
Let

$$
\phi(z)=\left(\phi_{2} \circ \phi_{1}\right)(z)=\frac{z^{2}-i}{z^{2}+i}: W \rightarrow \mathbb{D} \text { so that } \phi_{1}(z)=z^{2}
$$

If maps the infinite sector onto upper half plane $\mathbb{H}^{+}$and $\phi_{2}(w)=\frac{w-i}{w+i}$ maps the upper half-plane $\mathbb{H}^{+}$onto unit disk $\mathbb{D}$ (see Figure 5). Then the integrability of the derivative of conformal mapping $\phi$, is as follows:

$$
\iint_{W}\left|\phi^{\prime}(z)\right|^{p} d x d y<\infty ; \text { for each } p>\frac{2}{3}
$$

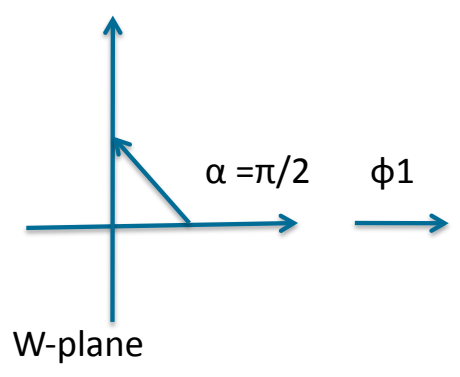

W-plane

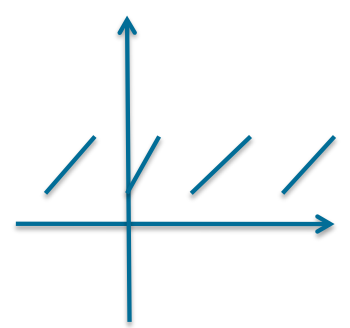

$\mathrm{H}+$-plane

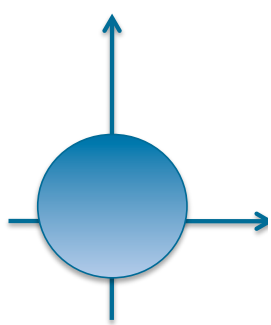

unit disk

FiguRE 5. Infinite sector $W$ for the angle $\alpha=\frac{\pi}{2}$

Proof. Given

$$
\begin{aligned}
\phi(z) & =\frac{z^{2}-i}{z^{2}+i}: W \rightarrow \mathbb{D} \\
\Rightarrow \phi^{\prime}(z) & =\frac{\left(z^{2}+i\right)(2 z)-\left(z^{2}-i\right)(2 z)}{\left(z^{2}+i\right)^{2}} \\
& =\frac{i 4 z}{\left(z^{2}+i\right)^{2}} \\
\Rightarrow\left|\phi^{\prime}(z)\right| & =\frac{4|z|}{\left|z^{2}+i\right|^{2}} .
\end{aligned}
$$

Now, $\mathbb{W}$-plane is an infinite sector. that is; $r=|z| \rightarrow 0-\infty$.

(i) so, $r=|z| \rightarrow \infty$ (i.e; $|z|$ is large). We know that

$$
\begin{aligned}
\left|z^{2}+i\right| & \geq|z|^{2}-1 \geq \frac{1}{2}|z|^{2} \\
\Rightarrow & \frac{1}{\left|z^{2}+i\right|} \leq \frac{2}{|z|^{2}} \\
\Rightarrow \frac{1}{\left|z^{2}+i\right|^{2}} & \leq \frac{4}{|z|^{4}}
\end{aligned}
$$


refer to the behaviour of $\left|z^{2}+i\right|$ at $\infty$ with respect to the region.

$$
\Rightarrow\left|\phi^{\prime}(z)\right|=\frac{4|z|}{\left|z^{2}+i\right|^{2}} \leq \frac{16|z|}{|z|^{4}}=16|z|^{-3}
$$

(ii) and $\mathbf{r}=|\mathbf{z}| \sim \mathbf{0}$

$$
\begin{gathered}
\Rightarrow\left|\phi^{\prime}(z)\right|=\frac{4|z|}{\left|z^{2}+i\right|^{2}} \Rightarrow\left|\phi^{\prime}(z)\right|=\frac{4|z|}{|i|^{2}}=4|z| . \\
\Rightarrow\left|\phi^{\prime}(z)\right| \leq\left\{\begin{array}{rr}
16|z|^{-3} & ;|z| \text { is large } \\
4|z| & ;|z| \sim 0
\end{array}\right. \\
\int_{0}^{\frac{\pi}{2}} \int_{0}^{\infty}\left|\phi^{\prime}\right|^{p} d x d y \leq 4 \int_{0}^{\frac{\pi}{2}} \int_{0}^{1}|z|^{p} r d r d \theta+16 \int_{0}^{\frac{\pi}{2}} \int_{1}^{\infty}|z|^{-3 p} r d r d \theta \\
\int_{0}^{\frac{\pi}{2}} \int_{0}^{\infty}\left|\phi^{\prime}\right|^{p} d x d y=2 \pi\left[\int_{0}^{1} r^{p+1} d r\right]+8 \pi\left[\int_{1}^{\infty} r^{-3 p+1} d r\right] . \\
\Rightarrow \int_{0}^{\frac{\pi}{2}} \int_{0}^{\infty}\left|\phi^{\prime}\right|^{p} d x d y \leq 2 \pi\left[\left.\frac{r^{p+2}}{p+2}\right|_{0} ^{1}+2 \pi\left[\left.\frac{r^{-3 p+2}}{-3 p+2}\right|_{1} ^{\infty}\right.\right.
\end{gathered}
$$

There are two definite integrals on the right-hand side of inequality (2.10).

The first one is clearly finite, and the second one is:

$$
-3 p+2<0 \Rightarrow-3 p<-2 \Rightarrow p>\frac{2}{3}
$$

Theorem 2.6. Let $\phi(z)$ be a conformal mapping defined on infinite sector $\mathbb{W}$ onto unit disk $\mathbb{D}$.

Let

$$
\phi(z)=\left(\phi_{2} \circ \phi_{1}\right)(z)=\frac{z^{4}-i}{z^{4}+i}: W \rightarrow \mathbb{D}
$$

such that $\phi_{1}(z)=z^{4}$ maps the infinite sector onto upper half plane $\mathbb{H}^{+}$and $\phi_{2}(w)=\frac{w-i}{w+i}$ maps the upper half plane $\mathbb{H}^{+}$onto unit disk $\mathbb{D}$ (see Figure 6). then the integrability of the derivative of conformal mapping is:

$$
\iint_{W}\left|\phi^{\prime}(z)\right|^{p} d x d y<\infty ; \text { for each } p>\frac{2}{5}
$$



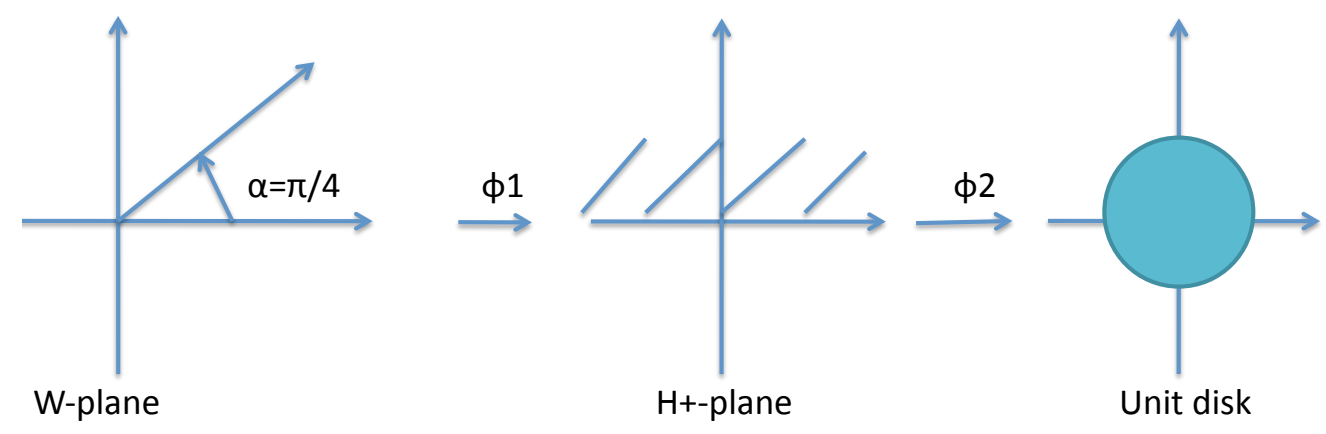

Figure 6 . Infinite sector $W$ for the angle $\alpha=\frac{\pi}{4}$

\section{Proof. Given}

$$
\begin{aligned}
\phi(z) & =\frac{z^{4}-i}{z^{4}+i}: W \rightarrow \mathbb{D} \\
\Rightarrow \phi^{\prime}(z) & =\frac{\left(z^{4}+i\right)\left(4 z^{3}\right)-\left(z^{4}-i\right)\left(4 z^{3}\right)}{\left(z^{4}+i\right)^{2}} \\
& =\frac{i 8 z^{3}}{\left(z^{4}+i\right)^{2}} \\
\Rightarrow\left|\phi^{\prime}(z)\right| & =\frac{8|z|^{3}}{\left|z^{4}+i\right|^{2}} .
\end{aligned}
$$

Now, W-plane is an infinite sector. that is; $r=|z| \rightarrow 0-\infty$.

- so,when $r=|z| \rightarrow \infty$ (that is; $|z|$ be large). We know that

$$
\begin{aligned}
\left|z^{4}+i\right| & \geq|z|^{4}-1 \geq \frac{1}{2}|z|^{4} \\
\Rightarrow & \frac{1}{\left|z^{4}+i\right|} \leq \frac{2}{|z|^{4}} \\
\Rightarrow \frac{1}{\left|z^{4}+i\right|^{2}} & \leq \frac{4}{|z|^{8}}
\end{aligned}
$$

This is referring to the behaviour of $\left|z^{4}+i\right|$ at $\infty$ with respect to the region.

$$
\Rightarrow\left|\phi^{\prime}(z)\right|=\frac{8|z|^{3}}{\left|z^{4}+i\right|^{2}} \leq \frac{8|z|^{3}}{|z|^{8}}=32|z|^{-5} \text {. }
$$

- and when $\mathbf{r}=|\mathbf{z}| \sim \mathbf{0}$

$$
\begin{gathered}
\Rightarrow\left|\phi^{\prime}(z)\right|=\frac{8|z|^{3}}{\left|z^{4}+i\right|^{2}} \Rightarrow\left|\phi^{\prime}(z)\right|=\frac{8|z|^{3}}{|i|^{2}}=8|z|^{3} . \\
\Rightarrow\left|\phi^{\prime}(z)\right| \leq\left\{\begin{array}{cl}
8|z|^{-5} & ;|z| \text { is large } \\
8|z|^{3} & ;|z| \sim 0
\end{array}\right.
\end{gathered}
$$




$$
\begin{aligned}
& \int_{0}^{\frac{\pi}{4}} \int_{0}^{\infty}\left|\phi^{\prime}\right|^{p} d x d y \leq 8 \int_{0}^{\frac{\pi}{4}} \int_{0}^{1} r^{3 p} r d r d \theta+8 \int_{0}^{\frac{\pi}{4}} \int_{1}^{\infty} r^{-5 p} r d r d \theta \\
&=2 \pi\left[\int_{0}^{1} r^{3 p+1} d r\right]+2 \pi\left[\int_{1}^{\infty} r^{-5 p+1} d r\right] \\
& \Rightarrow \int_{0}^{\frac{\pi}{4}} \int_{0}^{\infty}\left|\phi^{\prime}\right|^{p} d x d y \leq 2 \pi\left[\left.\frac{r^{3 p+2}}{3 p+2}\right|_{0} ^{1}+2 \pi\left[\left.\frac{r^{-5 p+2}}{-5 p+2}\right|_{1} ^{\infty}\right.\right.
\end{aligned}
$$

The first term on the right -hand- side of (2.11) is finite, and the second one is:

$$
-5 p+2<0 \Rightarrow-5 p<-2 \Rightarrow p>\frac{2}{5} .
$$

Theorem 2.7. Let $\phi(z)$ be a conformal mapping defined on infinite sector $\mathbb{W}$ onto unit disk $\mathbb{D}$.

Let

$$
\phi(z)=\left(\phi_{2} \circ \phi_{1}\right)(z)=\frac{z^{n}-i}{z^{n}+i}: W \rightarrow \mathbb{D}
$$

such that $\phi_{1}(z)=z^{n}$ maps the infinite sector onto upper half- plane $\mathbb{H}^{+}$where $\alpha$ is of the form $\alpha=\frac{\pi}{n}$ for some integer $n$ and $\phi_{2}(w)=\frac{w-i}{w+i}$ maps the upper half- plane $\mathbb{H}^{+}$onto unit disk $\mathbb{D}$ (see Figure 7 ). Then the integrability of the derivative of conformal mapping is as follows:

$$
\iint_{W}\left|\phi^{\prime}(z)\right|^{p} d x d y<\infty ; \text { for each } p>\frac{2}{\frac{3 \pi}{\alpha}+1} .
$$
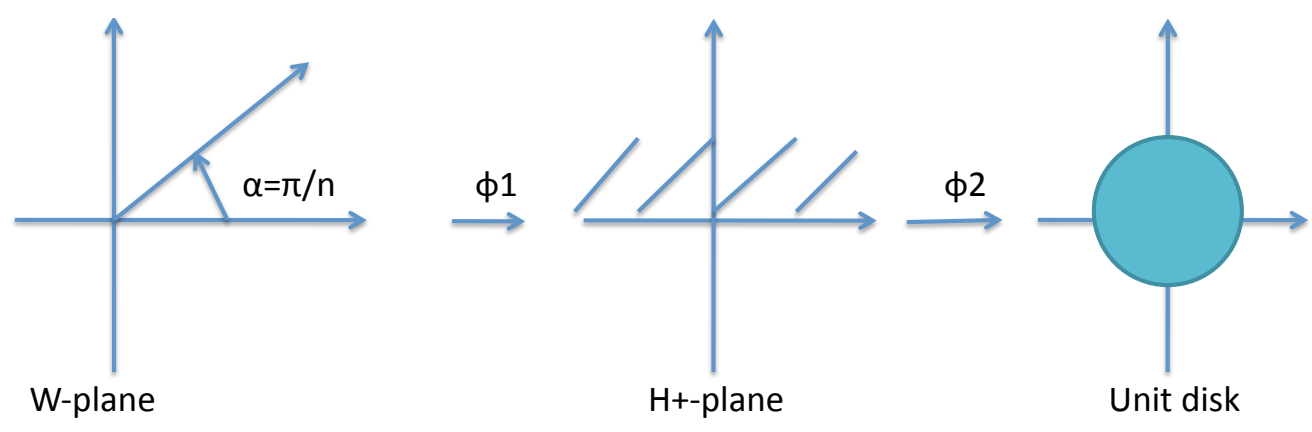

FiguRE 7. Infinite sector $W$ for the angle $\alpha=\frac{\pi}{n}$

Proof. Let $\alpha=\frac{\pi}{n}$ be the angle of the infinite sector $W$ which is mapped by $\phi_{1}=z^{n}$ onto upper half plane $\mathbb{H}^{+}$.

One can write $\phi_{1}=z^{\frac{\pi}{\alpha}}$.

We define the power function $\phi_{1}=z^{\frac{\pi}{\alpha}}$ to be the multivalued function

$$
z^{\frac{\pi}{\alpha}}=e^{\frac{\pi}{\alpha} \log z} ; z \neq 0
$$




$$
\begin{aligned}
\Rightarrow z^{\frac{\pi}{\alpha}} & =e^{\frac{\pi}{\alpha} \log |z|+i a r g z} \\
& =r^{\frac{\pi}{\alpha}} e^{i \frac{\pi}{\alpha} \theta} e^{ \pm i \frac{2 \pi^{2}}{\alpha} k}
\end{aligned}
$$

Various values of $z^{\frac{\pi}{\alpha}}$ are obtained from the principal value $e^{\frac{\pi}{\alpha} \log z}$ by multiplying by the integral power $\left(e^{i \frac{2 \pi^{2}}{\alpha}}\right)^{k}$ of $e^{i \frac{2 \pi^{2}}{\alpha}}$.

Let $\alpha=\frac{\pi}{n}$ is a number for some integer $n$, then the integral powers $e^{i \frac{2 \pi^{2}}{\alpha} k}$ of $e^{i \frac{2 \pi^{2}}{\alpha}}$ are exactly the $n$th roots of unity, and the values of $z^{\frac{\pi}{\alpha}}$ are the $n n$th roots of $z$.

$$
\begin{aligned}
\Rightarrow \phi(z) & =\frac{z^{\frac{\pi}{\alpha}}-i}{z^{\frac{\pi}{\alpha}}+i} \\
& =\frac{r^{\frac{\pi}{\alpha}} e^{i \frac{\pi}{\alpha}}-i}{r^{\frac{\pi}{\alpha}} e^{i \frac{\pi}{\alpha}}+i} \\
\phi(z) & =\frac{r^{\frac{\pi}{\alpha}} \cos \frac{\pi}{\alpha} \theta+i r^{\frac{\pi}{\alpha}} \sin \frac{\pi}{\alpha} \theta-i}{r^{\frac{\pi}{\alpha}} \cos \frac{\pi}{\alpha} \theta+i r^{\frac{\pi}{\alpha}} \sin \frac{\pi}{\alpha} \theta+i} .
\end{aligned}
$$

Simplify last equation (2.12) we get:

$$
\begin{aligned}
\phi(z) & =\frac{r^{\frac{2 \pi}{\alpha}}-2 i r^{\frac{\pi}{\alpha}} \cos \frac{\pi}{\alpha} \theta-1}{r^{\frac{2 \pi}{\alpha}}+2 r^{\frac{\pi}{\alpha}} \sin \frac{\pi}{\alpha} \theta+1} \\
& =\frac{r^{\frac{2 \pi}{\alpha}}-1}{r^{\frac{2 \pi}{\alpha}}+2 r^{\frac{\pi}{\alpha}} \sin \frac{\pi}{\alpha} \theta+1}+i \frac{-2 r^{\frac{\pi}{\alpha}} \cos \frac{\pi}{\alpha} \theta}{r^{\frac{2 \pi}{\alpha}}+2 r^{\frac{\pi}{\alpha}} \sin \frac{\pi}{\alpha} \theta+1} .
\end{aligned}
$$

When $\theta=0$ or $\theta=2 \pi$, it implies that:

$$
\begin{aligned}
\phi(z) & =\frac{r^{\frac{2 \pi}{\alpha}}-1}{r^{\frac{2 \pi}{\alpha}}+1}+i \frac{-2 r^{\frac{\pi}{\alpha}}}{r^{\frac{2 \pi}{\alpha}}+1} \\
& =\frac{r^{\frac{2 \pi}{\alpha}}-1-2 i r^{\frac{\pi}{\alpha}}}{r^{\frac{2 \pi}{\alpha}}+1}
\end{aligned}
$$

The derivative of $\phi(z)$ can be calculated:

$$
\begin{aligned}
& \phi^{\prime}(z)=\frac{\left(r^{\frac{2 \pi}{\alpha}}+1\right)\left[\frac{2 \pi}{\alpha} r^{\frac{2 \pi}{\alpha}-1}-2 i \frac{\pi}{\alpha} r^{\frac{\pi}{\alpha}-1}\right]-\left(r^{\frac{2 \pi}{\alpha}}-1-2 i r^{\frac{\pi}{\alpha}}\right)\left[\frac{2 \pi}{\alpha} r^{\frac{2 \pi}{\alpha}-1}\right]}{\left(r^{\frac{2 \pi}{\alpha}}+1\right)^{2}} \\
& =\frac{2 i \frac{\pi}{\alpha} r^{\frac{3 \pi}{\alpha}-1}+\frac{4 \pi}{\alpha} r^{\frac{4 \pi}{\alpha}-1}-2 i \frac{\pi}{\alpha} r^{\frac{\pi}{\alpha}-1}}{\left(r^{\frac{2 \pi}{\alpha}}+1\right)^{2}} \\
& =\frac{\frac{4 \pi}{\alpha} r^{\frac{4 \pi}{\alpha}-1}+i \frac{2 \pi}{\alpha}\left(r^{\frac{3 \pi}{\alpha}-1}-r^{\frac{\pi}{\alpha}-1}\right)}{\left(r^{\frac{2 \pi}{\alpha}}+1\right)^{2}} \\
& \left|\phi^{\prime}(z)\right|=\frac{\left|\frac{4 \pi}{\alpha} r^{\frac{4 \pi}{\alpha}-1}+i \frac{2 \pi}{\alpha}\left(r^{\frac{3 \pi}{\alpha}-1}-r^{\frac{\pi}{\alpha}-1}\right)\right|}{\left(r^{\frac{2 \pi}{\alpha}}+1\right)^{2}} \\
& \left|\phi^{\prime}(z)\right|=\frac{\sqrt{\frac{16 \pi^{2}}{\alpha^{2}} r^{\frac{8 \pi}{\alpha}-2}+\frac{4 \pi^{2}}{\alpha^{2}}\left(r^{\frac{3 \pi}{\alpha}-1}-r^{\frac{\pi}{\alpha}-1}\right)^{2}}}{\left(r^{\frac{2 \pi}{\alpha}}+1\right)^{2}}
\end{aligned}
$$


Again, W-plane is an infinite sector. that is; $|z| \rightarrow 0-\infty$.

(i) In case $r=|z| \rightarrow \infty$ (that is; $|z|$ be large).

$$
\left|\phi^{\prime}(z)\right|=\frac{\sqrt{\frac{16 \pi^{2}}{\alpha^{2}}|z|^{\frac{8 \pi}{\alpha}-2}+\frac{4 \pi^{2}}{\alpha^{2}}\left(|z|^{\frac{3 \pi}{\alpha}-1}-|z|^{\frac{\pi}{\alpha}-1}\right)^{2}}}{\left(|z|^{\frac{2 \pi}{\alpha}}+1\right)^{2}} .
$$

We know that,

$$
\begin{aligned}
|z|^{\frac{2 \pi}{\alpha}}+1 & \geq\left|z^{\frac{2 \pi}{\alpha}}+1\right| \geq|z|^{\frac{2 \pi}{\alpha}}-1 \geq \frac{1}{2}|z|^{\frac{2 \pi}{\alpha}} \\
\Rightarrow|z|^{\frac{2 \pi}{\alpha}}+1 & \geq \frac{1}{2}|z|^{\frac{2 \pi}{\alpha}} \\
\Rightarrow & \frac{1}{\left(|z|^{\frac{2 \pi}{\alpha}}+1\right)^{2}} \leq \frac{4}{|z|^{\frac{4 \pi}{\alpha}}} .
\end{aligned}
$$

Hence,

$$
\begin{aligned}
& \left|\phi^{\prime}(z)\right|=\frac{\sqrt{\frac{16 \pi^{2}}{\alpha^{2}}}|z|^{\frac{8 \pi}{\alpha}-2}+\frac{4 \pi^{2}}{\alpha^{2}}\left(|z|^{\frac{3 \pi}{\alpha}-1}-|z|^{\frac{\pi}{\alpha}-1}\right)^{2}}{\left(|z|^{\frac{2 \pi}{\alpha}}+1\right)^{2}} \leq \frac{4 \sqrt{\frac{16 \pi^{2}}{\alpha^{2}}|z|^{\frac{8 \pi}{\alpha}-2}+\frac{4 \pi^{2}}{\alpha^{2}}\left(|z|^{\frac{3 \pi}{\alpha}-1}-|z|^{\frac{\pi}{\alpha}-1}\right)^{2}}}{|z|^{\frac{4 \pi}{\alpha}}} \\
& \left|\phi^{\prime}(z)\right| \leq \frac{4 \sqrt{\frac{16 \pi^{2}}{\alpha^{2}} r^{\frac{8 \pi}{\alpha}-2}+\frac{4 \pi^{2}}{\alpha^{2}}\left(r^{\frac{3 \pi}{\alpha}-1}-r^{\frac{\pi}{\alpha}-1}\right)^{2}}}{r^{\frac{4 \pi}{\alpha}}}=\frac{4 \sqrt{\frac{16 \pi^{2}}{\alpha^{2}} r^{\frac{8 \pi}{\alpha}-2}+\frac{4 \pi^{2}}{\alpha^{2}} r^{\frac{2 \pi}{\alpha}-2}\left(r^{\frac{2 \pi}{\alpha}}-1\right)^{2}}}{r^{\frac{4 \pi}{\alpha}}} \\
& =\frac{4 \sqrt{\frac{4 \pi^{2}}{\alpha^{2}} r^{\frac{2 \pi}{\alpha}-2}\left[4 r^{\frac{6 \pi}{\alpha}}+\left(r^{\frac{2 \pi}{\alpha}}-1\right)^{2}\right]}}{r^{\frac{4 \pi}{\alpha}}} \\
& \left|\phi^{\prime}(z)\right| \leq \frac{4 \sqrt{\frac{4 \pi^{2}}{\alpha^{2}} r^{\frac{2 \pi}{\alpha}-2}\left[4 r^{\frac{6 \pi}{\alpha}}+\left(r^{\frac{2 \pi}{\alpha}}-1\right)^{2}\right]}}{r^{\frac{4 \pi}{\alpha}}}=\frac{\frac{8 \pi}{\alpha} r^{\frac{\pi}{\alpha}-1} \sqrt{4 r^{\frac{6 \pi}{\alpha}}+\left(r^{\frac{2 \pi}{\alpha}}-1\right)^{2}}}{r^{\frac{4 \pi}{\alpha}}} \\
& =\frac{8 \pi}{\alpha} r^{\frac{-3 \pi}{\alpha}-1} \sqrt{4 r^{\frac{6 \pi}{\alpha}}+\left(r^{\frac{2 \pi}{\alpha}}-1\right)^{2}}
\end{aligned}
$$

(ii) In case $\mathbf{r}=|\mathbf{z}| \sim \mathbf{0}$

$$
\begin{aligned}
\left|\phi^{\prime}(z)\right| & =\sqrt{\frac{16 \pi^{2}}{\alpha^{2}}|z|^{\frac{8 \pi}{\alpha}-2}+\frac{4 \pi^{2}}{\alpha^{2}}\left(|z|^{\frac{3 \pi}{\alpha}-1}-|z|^{\frac{\pi}{\alpha}-1}\right)^{2}} . \\
\Rightarrow\left|\phi^{\prime}(z)\right| & =\sqrt{\frac{16 \pi^{2}}{\alpha^{2}} r^{\frac{8 \pi}{\alpha}-2}+\frac{4 \pi^{2}}{\alpha^{2}}\left(r^{\frac{3 \pi}{\alpha}-1}-r^{\frac{\pi}{\alpha}-1}\right)^{2}} \\
& =\sqrt{\frac{16 \pi^{2}}{\alpha^{2}} r^{\frac{8 \pi}{\alpha}-2}+\frac{4 \pi^{2}}{\alpha^{2}} r^{\frac{2 \pi}{\alpha}-2}\left(r^{\frac{2 \pi}{\alpha}}-1\right)^{2}} \\
& =\sqrt{\frac{4 \pi^{2}}{\alpha^{2}} r^{\frac{2 \pi}{\alpha}-2\left[4 r^{\frac{6 \pi}{\alpha}}+\left(r^{\frac{2 \pi}{\alpha}}-1\right)^{2}\right]}} \\
& =\frac{2 \pi}{\alpha} r^{\frac{\pi}{\alpha}-1} \sqrt{4 r^{\frac{6 \pi}{\alpha}}+\left(r^{\frac{2 \pi}{\alpha}}-1\right)^{2}} .
\end{aligned}
$$


In the end,

$$
\begin{aligned}
& \Rightarrow\left|\phi^{\prime}(z)\right| \leq\left\{\begin{array}{cl}
\frac{8 \pi}{\alpha} r^{\frac{-3 \pi}{\alpha}-1} \sqrt{4 r^{\frac{6 \pi}{\alpha}}+\left(r^{\frac{2 \pi}{\alpha}}-1\right)^{2}} & ;|z| \text { is large } \\
\frac{2 \pi}{\alpha} r^{\frac{\pi}{\alpha}-1} \sqrt{4 r^{\frac{6 \pi}{\alpha}}+\left(r^{\frac{2 \pi}{\alpha}}-1\right)^{2}} . & ;|z| \sim 0
\end{array}\right. \\
& \int_{0}^{\frac{\pi}{n}} \int_{0}^{\infty}\left|\phi^{\prime}(z)\right|^{p} d x d y \leq \int_{0}^{\frac{\pi}{n}} \int_{0}^{1}\left(\frac{2 \pi}{\alpha}\right)^{p} r^{\left(\frac{\pi}{\alpha}-1\right) p}\left[4 r^{\frac{6 \pi}{\alpha}}+\left(r^{\frac{2 \pi}{\alpha}}-1\right)^{2}\right]^{\frac{p}{2}} \cdot r d r d \theta \\
& +\int_{0}^{\frac{\pi}{n}} \int_{1}^{\infty}\left(\frac{8 \pi}{\alpha}\right)^{p} r^{\left(\frac{-3 \pi}{\alpha}-1\right) p}\left[4 r^{\frac{6 \pi}{\alpha}}+\left(r^{\frac{2 \pi}{\alpha}}-1\right)^{2}\right]^{\frac{p}{2}} r d r d \theta \\
& \int_{0}^{\frac{\pi}{n}} \int_{0}^{\infty}\left|\phi^{\prime}(z)\right|^{p} d x d y \leq\left(\frac{2 \pi}{\alpha}\right)^{p}\left(\frac{\pi}{n}\right) \int_{0}^{1} r^{\left(\frac{\pi}{\alpha}-1\right) p+1} d r \int_{0}^{1}\left[4 r^{\frac{6 \pi}{\alpha}}+\left(r^{\frac{2 \pi}{\alpha}}-1\right)^{2}\right]^{\frac{p}{2}} \cdot r d r \\
& +\left(\frac{8 \pi}{\alpha}\right)^{p}\left(\frac{\pi}{n}\right) \int_{1}^{\infty} r^{\left(\frac{-3 \pi}{\alpha}-1\right) p+1} d r \int_{1}^{\infty}\left[4 r^{\frac{6 \pi}{\alpha}}+\left(r^{\frac{2 \pi}{\alpha}}-1\right)^{2}\right]^{\frac{p}{2}} d r .
\end{aligned}
$$

such that, we have four terms. The first, second and fourth terms on the right -hand-side of (2.4) becomes finite only when

$$
\begin{aligned}
& \left(\frac{-3 \pi}{\alpha}-1\right) p+2 \leq 0 \Rightarrow\left(\frac{-3 \pi}{\alpha}-1\right) p \leq-2 \\
& \Rightarrow-\left(\frac{3 \pi}{\alpha}+1\right) p \leq-2 \\
& \Rightarrow\left(\frac{3 \pi}{\alpha}+1\right) p \geq 2 \\
& \Rightarrow p>\frac{2}{\frac{3 \pi}{\alpha}+1} .
\end{aligned}
$$

Acknowledgment: The author would like to express a deep thanks and gratitude to Department of Mathematics, College of Science, Mustansiriyah University for deep supporting to appear this research paper as it is now.

Conflicts of Interest: The author(s) declare that there are no conflicts of interest regarding the publication of this paper.

\section{REFERENCES}

[1] S. S. Alhily, Higher Integrability of the Gradient of Conforaml Maps, PhD thesis, University of Sussex, 2013.

[2] S. S. Alhily and Deepmala, Boundary Behaviour of Holomorphic Functions on the Cardioid Domain with some Applications, Bol. Soc. Paran. Mat. 38(7) (2020), 203-218.

[3] P. L. Duren, Univalent Functions, third ed., Springer-Verlag, New York, 1983.

[4] P. H. Difrancesco, P. Mathieu, D. Sénéchal, Conformal Field Theory, first ed., Springer-Verlag Berlin Heidelberg, New York, 1997. 
[5] T. A. Driscoll, L. N. Trefethen, Schwarz-Christoffel Mapping. Cambridge Monographs on Applied and Computational Mathematics, vol. 8. Cambridge University Press, Cambridge, 2002.

[6] S. G. Krantz, Complex Analysis: The Geometric Viewpoint, vol.23, Mathematical Association of America, Washington, D.C., 1990.

[7] V.N. Mishra and G. Tomar, Exiistance of wandering and periodic domain in given angular region, Math. Slovaca, 70(4) (2020), 839-848.

[8] Ch. Pommerenke, Univalent functions, first ed., Vandenhoeck and Ruprecht, Göttingen, Germany, 1975.

[9] Ch. Pommerenke, Boundary Behaviour of Conformal Mappings, second ed., Springer-Verlag Berlin Heidelberg, 1992.

[10] E. M. Stein, R. Shakarchi, Complex Analysis. Princeton University Press, Princeton, 2003.

[11] H. A. Saleh, S. S. Alhily, Growth and Bounded Solution of Second-Order of Complex Differential Equations Through of Coefficient Function, IOP Conf. Ser.: Mater. Sci. Eng. 871 (2020) 012057.

[12] G. Tomar and V.N. Mishra, Maximum term of transcendental entire function and Spider's web, Math. Slovaca, 70(4) (2020), 81-86. 